\title{
Focal eosinophilic myositis presenting with leg pain and tenderness
}

\author{
Jin-Hong Shin ${ }^{1,2}$, Dae-Seong Kim ${ }^{1,2}$ \\ 'Department of Neurology, Research Institute for Convergence of Biomedical Research, Pusan National University \\ Yangsan Hospital, Yangsan, Korea \\ ${ }^{2}$ Department of Neurology, Pusan National University School of Medicine, Yangsan, Korea
}

Received: September 11, 2020

Revised: September 29, 2020

Accepted: September 29, 2020

\section{Correspondence to}

\section{Dae-Seong Kim}

Department of Neurology, Pusan National Univeristy School of Medicine, 20 Geumo-ro, Mulgeum-eup, Yangsan 50612, Korea

Tel: +82-55-360-2450

Fax: +82-55-360-2152

E-mail:dskim@pusan.ac.kr

ORCID

\section{Jin-Hong Shin}

https://orcid.org/0000-0002-5174-286X

Dae-Seong Kim

https://orcid.org/0000-0003-0586-4775
Focal eosinophilic myositis (FEM) is the most limited form of eosinophilic myositis that commonly affects the muscles of the lower leg without systemic manifestations. We report a patient with FEM who was studied by magnetic resonance imaging and muscle biopsy with a review of the literature.

Key words: Myositis; Eosinophils; Magnetic resonance imaging

Eosinophilic myositis (EM) is defined as a group of idiopathic inflammatory myopathies associated with peripheral and/or intramuscular eosinophilia. ${ }^{1}$ Focal eosinophilic myositis (FEM) is the most limited form of EM and is considered a benign disorder without systemic manifestations. ${ }^{2}$ Here, we report a patient with localized leg pain and tenderness who was diagnosed as FEM based on laboratory findings, magnetic resonance imaging (MRI), and muscle biopsy.

\section{CASE}

A 26-year-old otherwise healthy man visited our outpatient clinic with leg pain for 3 months. He recalled that he had had a transient throat pain about 1 week before the onset of the leg pain. Initially, he developed bilateral calf pain with subsequent tenderness and mild swelling within a few days. The calf swelling persisted for a month and then spontaneously regressed for a few weeks. For 3 months, he had moderate calf pain on exercise and experienced a sense of weakness in his leg when walking. However, he was able to perform all his daily activities during his illness. He denied recent traumatic injury, infectious disease, atopy, or allergic disease. He had no previous history of diabetes, hypertension, or other chronic diseases, and he was not taking any medication or special diet.

On examination, neither focal muscle enlargement nor palpable masses were noted

\section{Copyright @ 2020 The Korean Society of Clinical Neurophysiology}

This is an Open Access article distributed under the terms of the Creative Commons Attribution Non-Commercial License (http:// creativecommons.org/licenses/by-nc/4.0) which permits unrestricted non-commercial use, distribution, and reproduction in any medium, provided the original work is properly cited. 
in the leg muscles, but he had significant tenderness in both calves. Skin lesions were not observed. Neurological examination was unremarkable, including individual muscle power, cognitive functions, cranial nerves, station and gait, sensory function, and deep tendon reflexes.

Complete blood count revealed elevated eosinophil count (1,200/ $\mu \mathrm{L}$; normal range: $0-500 / \mu \mathrm{L})$ and ratio (15.2\%; normal range: 0-6\%). Serum erythrocyte sedimentation rate (21 mm/h; normal range: 0-10 mm/h) and C-reactive protein (0.64 mg/\%; normal range: 0-0.5 mg/\%) levels were mildly elevated. Serum immunoglobulin E level was also elevated to $393.5 \mathrm{lU} / \mathrm{mL}$ (normal range: $<100 \mathrm{IU} / \mathrm{mL}$ ). These findings led us to examine the stool for parasite eggs and serum antibodies against Clonorchis, cysticercus, Paragonimus, and Sparganum, all of which were negative. Serum creatine kinase level was within normal limits (107 U/L; normal range: 0-171 U/L). Other laboratory tests, including rheumatoid factor, anti-nuclear antibody, liver and renal function tests, thyroid function tests, serum electrolytes, urinalysis, serum glucose, chest roentgenogram, and electrocardiogram were unremarkable. Needle electromyography showed positive sharp waves in the left gastrocnemius muscle with normal-sized motor unit potentials and recruitment patterns. The left tibialis anterior, vastus lateralis, gluteus medius, glu-
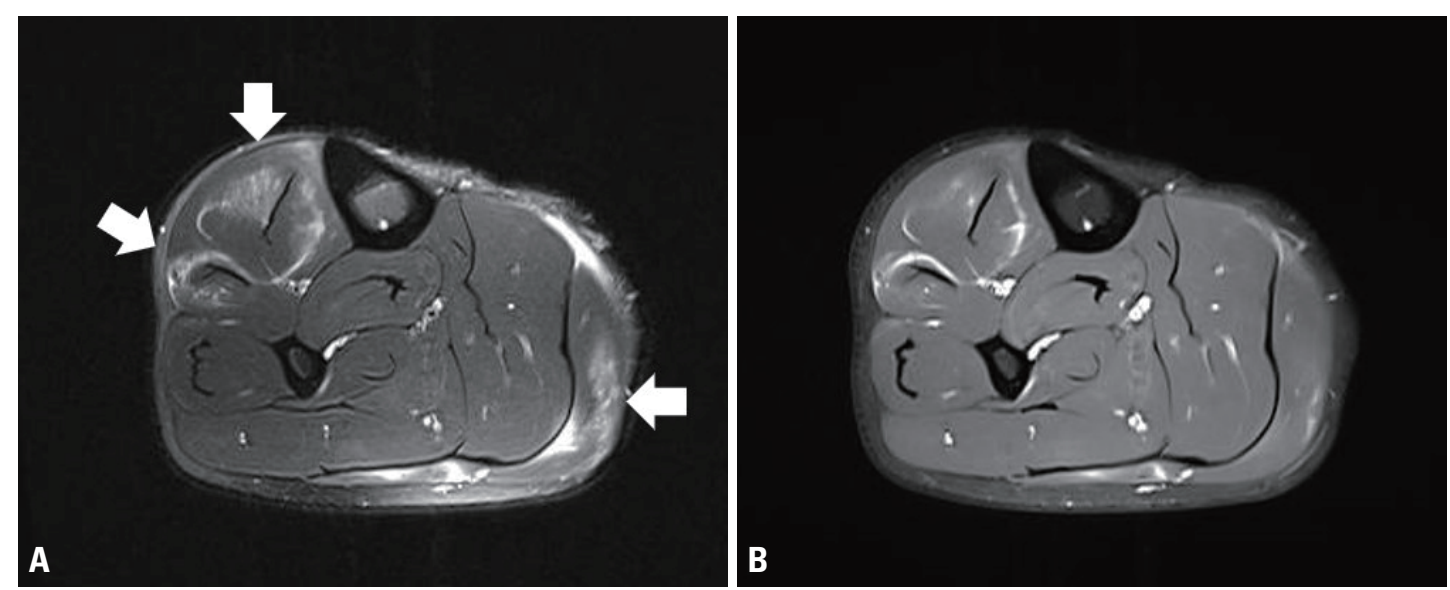

Fig. 1. T2-weighted muscle magnetic resonance imaging at the right mid-calf level shows multifocal high signal intensities in the tibialis anterior, extensor digitorum longus, and gastrocnemius muscles (A, white arrows). Gadolinium-enhanced T1-weighted image shows an enhancement at the same area (B).
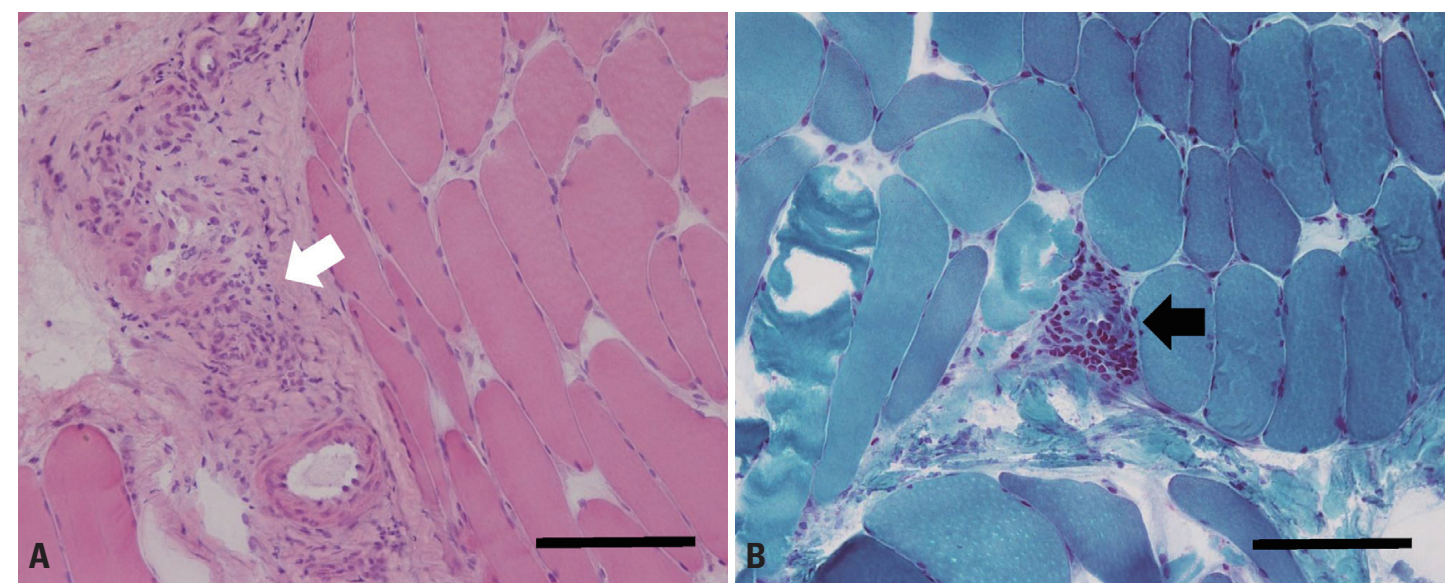

Fig. 2. Muscle biopsy from tibialis anterior muscle shows mononuclear cellular infiltrates in perimysial (A, white arrow) and perivascular (B, black arrow) spaces. Increased number of muscle fibers with internal nuclei was also observed (A). Black bar indicates $100 \mu$ m. (A) Hematoxylin and eosin stain ( $\times 200)$. (B) Modified Gomori-trichrome stain (x200). 
teus maximus, and lumbosacral paraspinal muscles showed normal electromyographic findings. Nerve conduction study performed in the bilateral lower extremity was within normal limits. MRI showed multifocal increased signal intensities in the right tibialis anterior, extensor digitorum longus, and gastrocnemius muscles. Gadolinium-enhanced T1-weighted images showed an enhancement in the same areas (Fig. 1). There were no MRI findings suggesting deep vein thrombosis, such as vessel distension, perivascular edema, or signal changes of the vessels. Muscle biopsy performed on the right tibialis anterior muscle showed inflammatory cellular infiltrates in the perivascular and perimysial areas. Cellular infiltrates mainly comprised mononuclear cells, and no definite eosinophils were observed (Fig. 2).

Based on the above findings, the patient was diagnosed with FEM affecting the lower extremity muscles. Because he did not show objective muscle weakness or systemic symptoms, symptomatic therapy using a non-steroidal anti-inflammatory agent was performed. After 3 months of follow-up, he did not complain of calf pain or tenderness, and laboratory values were normalized, including peripheral eosinophilia.

\section{DISCUSSION}

Eosinophilia is defined as an absolute eosinophil count in the peripheral blood of more than 500/ $\mu \mathrm{L}^{3}$. A few types of muscle disease can be associated with peripheral or tissue eosinophilia including eosinophilia-myalgia syndrome caused by L-tryptophan, parasitic myositis, Churg-Strauss syndrome, and idiopathic eosinophilic myositis or fasciitis. ${ }^{4}$ Idiopathic eosinophilic myositis is a clinically and pathologically heterogeneous entity that includes three main subtypes: FEM, eosinophilic polymyositis, and eosinophilic perimyositis. ${ }^{2}$

FEM is the most limited form of EM and is considered a benign disease without organ involvement or systemic manifestations. ${ }^{5}$ Muscle involvement in FEM is typically focal and circumscribed to the lower legs and usually manifests with leg pain, calf swelling, and tenderness, as observed in the present case. Muscle pathology shows perimysial and endomysial mononuclear cell infiltration with or without eosinophils, as well as muscle fiber necrosis and regeneration.
As observed in the present case, symptoms and laboratory abnormalities usually improve spontaneously without specific treatment. ${ }^{2}$ Because FEM can also present as localized painful nodular lesions in the lower extremities, localized nodular myositis should be differentiated based on laboratory and/or pathological findings. ${ }^{6}$

The present case satisfied two major criteria (pain and calf swelling and mononuclear cell infiltration [eosinophilic or not] with muscle cell invasion and necrosis on muscle biopsy) and three minor criteria (MRI or electromyographic evidence of focal myositis, absence of systemic illness, and peripheral eosinophilia) proposed by Selva-O'Callaghan et al. ${ }^{2}$, and was compatible with a diagnosis of FEM. Muscle MRI played an important role in reaching a diagnosis in the presented case because it showed direct evidence of muscle involvement providing important information on the extent and distribution of the lesion that led us to perform a diagnostic muscle biopsy. We regret that we did not perform ultrasound and D-dimer examination in order to exclude the possibility of deep vein thrombosis, which is the most important differential diagnosis of FEM. However, the presence of peripheral eosinophilia, a benign clinical course, and the absence of MRI findings suggesting venous thrombosis led us to reject the diagnosis of deep vein thrombosis. Recognition of this rare form of myositis will help clinicians to adopt a systematic approach and provide optimal treatment in patients with leg pain and swelling.

\section{Acknowledgements}

This study was supported by a 2-year (2019-2021) research grant from Pusan National University (Dae-Seong Kim).

\section{Conflicts of Interest}

The authors declare no conflicts of interest relevant to this article.

\section{REFERENCES}

1. Kaufman LD, Kephart GM, Seidman RJ, Buhner D, Qvarfordt I, Nässberger $L$, et al. The spectrum of eosinophilic myositis. Clinical and immunopathogenic studies of three patients, and review of the literature. Arthritis Rheum 1993;36:1014-1024.

2. Selva-O'Callaghan A, Trallero-Araguás E, Grau JM. Eosinophilic 
myositis: an updated review. Autoimmun Rev 2014;13:375-378.

3. Gotlib J. World Health Organization-defined eosinophilic disorders: 2017 update on diagnosis, risk stratification, and management. Am J Hematol 2017;92:1243-1259.

4. Trüeb RM, Pericin M, Winzeler B, Wüthrich B, Burge G. Eosinophilic myositis/perimyositis: frequency and spectrum of cutaneous manifestations. J Am Acad Dermatol 1997;37:385-391.

5. Kobayashi Y, Fujimoto T, Shiiki H, Kitaoka K, Murata K, Dohi K. Focal eosinophilic myositis. Clin Rheumatol 2001;20:369-371.

6. Matsuse D, Ikezoe K, Shigeto H, Murai H, Ohyagi Y, Kira J. Case of eosinophilic myositis in continuum from localized nodular myositis. Rinsho Shinkeigaku 2008;48:36-42. 